\title{
USO DO ESPAÇO POR PEQUENOS MAMÍFEROS: UMA ANÁLISE DOS ESTUDOS REALIZADOS NO BRASIL
}

\author{
Jayme A. Prevedello ${ }^{l,}{ }^{*}$, André F. Mendonça ${ }^{l}$ \& Marcus V. Vieira ${ }^{l}$ \\ 1. Laboratório de Vertebrados, Departamento de Ecologia, IB, Universidade Federal do Rio de Janeiro. Cidade Universitária, CEP 21941-590, Rio de \\ Janeiro, RJ, Brasil, Caixa Postal 68020. \\ *E-mail: ja_prevedello@yahoo.com.br.
}

\section{RESUMO}

$\mathrm{O}$ uso do espaço refere-se à quantidade, qualidade e intensidade de exploração do habitat em uma determinada localidade, sendo importante determinante da distribuição e abundância de espécies animais. Os pequenos mamíferos brasileiros têm sido utilizados como modelos para avaliar o uso do espaço por mamíferos tropicais, mas pouco se sabe sobre a representatividade do conhecimento obtido em termos de espécies, biomas, atributos abordados e técnicas utilizadas. Neste estudo, fizemos uma revisão da literatura publicada sobre o tema no período de 1945 a 2007. Foram encontrados 58 estudos, a maioria (65\%) destes realizada na Mata Atlântica. Neste bioma, marsupiais e roedores foram analisados em $86 \%$ e 53\% dos artigos respectivamente, mas no Cerrado essas proporções mudaram para 44\% e 100\%. Um pequeno número de gêneros de marsupiais e roedores concentra a maior parte das informações existentes sobre o uso do espaço. Somente $14 \%$ dos estudos avaliaram o uso vertical do espaço. A grande maioria (75\%) das publicações utilizou a técnica de captura-marcação-recaptura com armadilhas (CMR). O conhecimento atual sobre o uso do espaço é limitado por ser baseado principalmente em um pequeno número de gêneros de um único bioma (Mata Atlântica), em estudos que utilizaram uma técnica que não amostra grande parte da movimentação dos animais (CMR). Novos estudos que preencham as lacunas aqui apontadas podem permitir a detecção e o estabelecimento de padrões mais gerais de uso do espaço por mamíferos tropicais.

Palavras-chave: área de vida, seleção de habitat, estratificação vertical, rádio-telemetria, carretel de rastreamento.

\begin{abstract}
SPACE UTILIZATION BY SMALL MAMMALS: AN OVERVIEW OF STUDIES IN BRAZIL. Utilization of space refers to the intensity of exploitation of a habitat by a species, including the amount and quality of the exploited habitat. It is an important factor driving the abundance and distribution of animal species. Brazilian small mammals have been used as models to evaluate space utilization by tropical mammals in general, but it is yet unclear whether knowledge about the species, biomes, different aspects of space use, and even the techniques being employed in Brazil are representative of other regions. The present study presents a review of studies on space utilization by small mammals in Brazil published from 1945 to 2007. Fifty-eight publications were found, most of which were conducted in the Atlantic Forest (65\%). Within this biome, marsupials were the most common taxon examined ( $86 \%$ ) followed by rodents $(53 \%)$. In the Cerrado, these figures changed to $44 \%$ and $100 \%$, respectively. Only $14 \%$ of the studies dealt with vertical utilization of space. The great majority of the publications (75\%) used capture-mark-recapture methods with livetraps (CMR). Current knowledge of space utilization by Brazilian small mammals was mainly based on few genera from a single biome - the Atlantic Forest - using a technique that records only part of the movement patterns of these animals (CMR). Future studies that fill the gaps indicated here will hopefully allow the detection and establishment of more general patterns of space use by tropical mammals.
\end{abstract}

Keywords: home range, habitat selection, vertical stratification, radiotracking, spool-and-line technique. 


\section{INTRODUÇÃO}

O espaço físico é considerado uma das principais dimensões do nicho de uma espécie, uma vez que a maneira como é utilizado determina uma série de interações ecológicas com o ambiente (Schoener 1974, Pianka 1999). O uso do espaço influencia, por exemplo, a estrutura e dinâmica populacional (ex. Fernandez et al. 1997, Gentile \& Cerqueira 1995) e a estrutura das comunidades animais (ex. Cunha \& Vieira 2002, Grelle 2003, Vieira \& MonteiroFilho 2003), sendo talvez o mais importante fator na coexistência das espécies (Schoener 1974, Cunha \& Vieira 2004). Devido a sua relevância teórica, o uso do espaço tem uma longa história de estudo na ecologia animal, como exemplificado por estudos sobre seleção de habitat e padrões de movimentação (Lima \& Zollner 1996, Garshelis 2000).

Os mamíferos têm recebido grande atenção por parte dos ecólogos em estudos sobre o uso do espaço. Esse interesse se deve principalmente a um grupo em particular, os chamados pequenos mamíferos, que apresentam alta riqueza, densidade e variedade de hábitos (Fonseca et al. 1996, Emmons 1997) facilitando a obtenção de uma série de respostas ecológicas (Delany 1974). Esses fatores contribuem para que um grande volume de informações relativas à ecologia de pequenos mamíferos tenha sido coletado ao redor do mundo, incluindo informações sobre sua ocupação e utilização do espaço.
No Brasil, os pequenos mamíferos não-voadores $(<3 \mathrm{~kg})$ são representados pelas ordens Didelphimorphia (marsupiais), Rodentia (roedores) e Lagomorpha (coelhos) (Fonseca et al. 1996). As espécies das ordens Didelphimorphia e Rodentia têm sido usadas como modelos para o estudo do uso do espaço por mamíferos neotropicais. O tamanho corporal, os hábitos e, consequentemente, os métodos de captura para marsupiais e pequenos roedores brasileiros são similares, o que favorece seu estudo ecológico em conjunto. Uma vez que a maioria das espécies desses grupos apresenta hábitos noturnos e similaridades na dieta (Fonseca et al. 1996, Emmons 1997), a dimensão espacial do nicho se torna a mais importante na coexistência das espécies, o que ressalta a relevância do estudo do uso do espaço (Cunha \& Vieira 2002, 2004).

O interesse no uso do espaço por pequenos mamíferos brasileiros tem crescido acentuadamente, sendo que $50 \%$ dos estudos foram publicados nos últimos 10 anos (Figura 1). As poucas revisões relacionadas ao tema se restringem ao grupo dos marsupiais (Cáceres \& Monteiro-Filho 2006), sumarizando o conhecimento teórico obtido para apenas alguns dos atributos que compõem o uso do espaço. Não existem revisões sobre os conceitos abordados, técnicas utilizadas e biomas e grupos biológicos estudados.

Visando sumarizar o conhecimento atual e direcionar o foco de novos estudos, fizemos um levantamento da literatura publicada sobre o uso do espaço

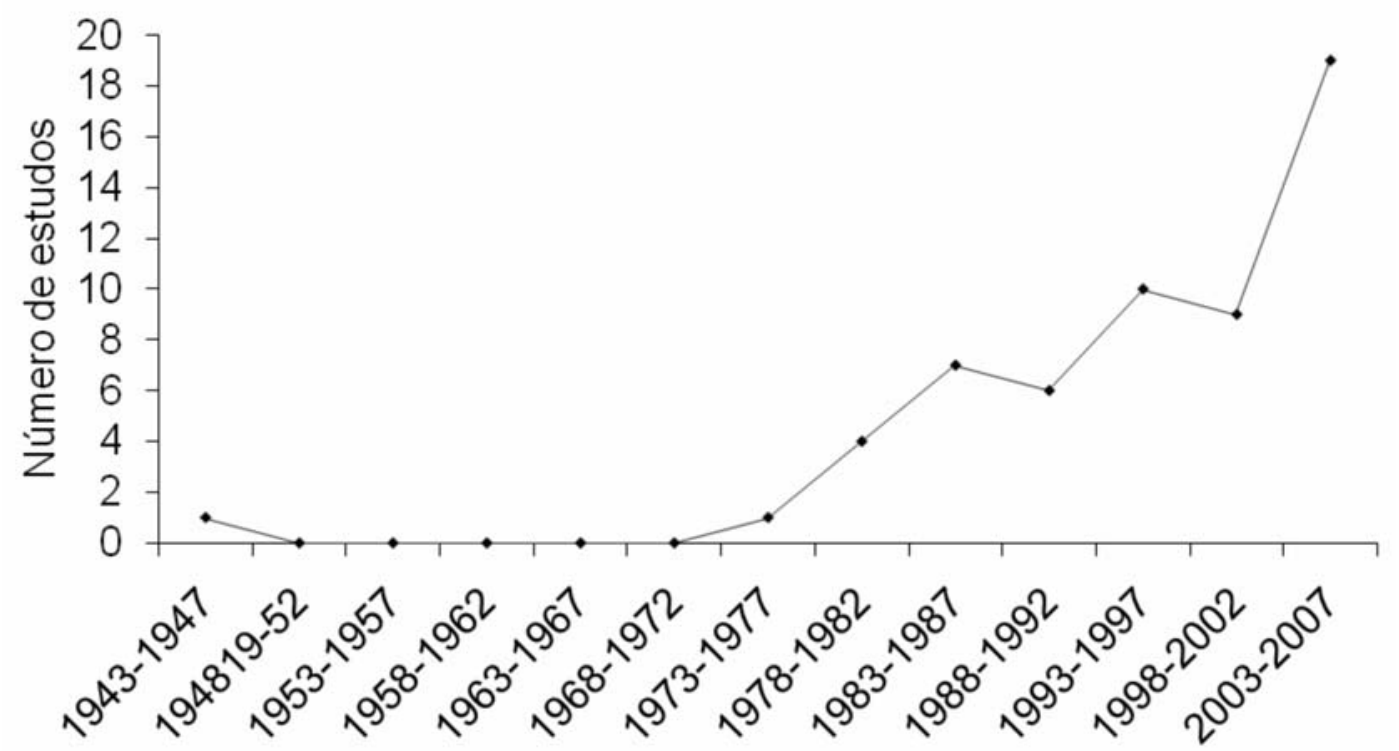

Figura 1. Evolução no número de estudos publicados no período de 1945-2007 sobre uso do espaço por pequenos mamíferos brasileiros.

Figure 1. Increase of the number of studies on space utilization by Brazilian small mammals published between 1945 and 2007. 
por pequenos mamíferos no Brasil. Dada a amplitude e a imprecisão conceitual do tema, apresentamos também uma breve revisão dos conceitos relacionados ao uso do espaço. As principais técnicas de estudo são também descritas para auxiliar o leitor na interpretação dos resultados.

\section{O QUE É "USO DO ESPAÇO”? DEFININDO ALGUNS CONCEITOS}

O conceito de uso do espaço é amplo e tem sido empregado na literatura para o estudo de diferentes características ecológicas. Muitos autores utilizam o termo como sinônimo de área de vida (ex. Jacob \& Rudran 2003), enquanto alguns trabalhos consideram que o uso do espaço engloba também os padrões de agregação espacial da população (Gentile \& Cerqueira 1995) e a estratificação vertical de uma comunidade (Vieira 2006). Uso do espaço e seleção de habitat são geralmente tratados como atributos diferentes e complementares (ex. Valenzuela \& Ceballos 2000), mas alguns trabalhos consideram a seleção de habitat parte integrante do uso do espaço (ex. Cáceres \& Monteiro-Filho 2006). Outros ainda não fazem distinção entre uso e seleção de habitat, considerando-os erroneamente como sinônimos. $\mathrm{Na}$ presente revisão, adotaremos uma definição abrangente do termo, considerando que o uso do espaço se refere à exploração do habitat em termos de quantidade, qualidade e intensidade em uma determinada localidade. Quantidade se refere à área ou volume de habitat utilizado por um indivíduo. Qualidade se refere ao local escolhido pelo animal para posicionar tal área, considerando-se a complexidade e heterogeneidade (sensu August 1983) de habitat existente. Engloba, assim, a seleção de habitat e a estratificação vertical da comunidade. A intensidade, por sua vez, se refere à distribuição das atividades individuais dentro da área de vida, à defesa ou não de tal área contra outros indivíduos (territorialismo) e a distribuição espacial da população como um todo em uma determinada área.

Assim definido, o uso do espaço englobaria não apenas características individuais, mas também populacionais e comunitárias, reunindo pelo menos sete atributos ecológicos (Tabela I). Todos eles se aplicam ao entendimento da ocupação, exploração e compartilhamento, em escala local, do espaço físico existente por um grupo de organismos (indivíduos, populações ou espécies). Uma breve definição de cada atributo é apresentada a seguir.

Tabela I. Atributos que compõem o uso do espaço e sua relação com os níveis hierárquicos de estudo da Ecologia.

Table I. Attributes of space use and their relationship with hierarchical levels in Ecology.

\begin{tabular}{ll}
\hline $\begin{array}{c}\text { Nível de abordagem } \\
\text { ecológica }\end{array}$ & Atributos relacionados \\
\hline Indivíduo & $\begin{array}{l}\text { Área de vida, mobilidade, } \\
\text { intensidade de uso vertical, } \\
\text { uso de abrigos }\end{array}$ \\
\hline População & $\begin{array}{l}\text { Seleção de habitat, padrão } \\
\text { de agregação espacial }\end{array}$ \\
\hline Comunidade & Estratificação vertical \\
\hline
\end{tabular}

a) Área de vida e território: A área de vida ("home range") é definida como a área utilizada por um indivíduo em suas atividades normais em busca de alimento, acasalamento e cuidado com a prole (Burt 1943). A área de vida é geralmente avaliada em duas dimensões do espaço, mas para animais voadores, arborícolas, escansoriais e aquáticos, deve ser quantificada tridimensionalmente (Meserve 1977, Koeppl et al. 1977, Grelle 1996). Já o território é uma área mais restrita, dentro da área de vida, que é defendida direta ou indiretamente contra outros indivíduos ou grupos (Noble 1939 apud Bergallo 1990). Recentemente, têm-se estudado a área utilizada em movimentos menores ou "área de vida diária" (daily home range, conforme Spencer et al. 1990) a qual representa uma medida em curto prazo espaço-temporal dos requerimentos espaciais de um indivíduo (Spencer et al. 1990, Loretto \& Vieira 2008, Vieira \& Cunha 2008). Uma das razões para esta recente ênfase na área de vida diária é a relação com o tamanho corporal, a qual difere da área de vida tradicional (Vieira \& Cunha 2008). A área de vida é estudada principalmente com uso de armadilhas de captura (ex. Alho \& Souza 1982, Magnusson et al. 1995, Gentile et al. 1997) e pela técnica de rádiotelemetria (Moraes Júnior \& Chiarello 2005a, Lira et al. 2007), enquanto que a área de vida diária pode ser avaliada também com carretéis de rastreamento (Miles et al. 1981, Loretto \& Vieira 2005).

b) Mobilidade: Se refere a medidas de taxa de movimentação (distância percorrida por unidade de tempo) ou extensão dos movimentos (distância 
máxima ou média percorrida pelo animal entre dois pontos de sua área de vida). Dois animais podem ter tamanhos de área de vida similares, mas taxas de movimentação bastante diferentes dentro dessa área. As medidas de mobilidade mais utilizadas são a distância entre recapturas ou entre duas localizações (rádio-telemetria) ou a distância máxima percorrida em um trajeto (carretel de rastreamento). As medidas de mobilidade são úteis, por exemplo, para detectar comportamentos exploratórios (ex. Gentile \& Cerqueira 1995), dispersão entre fragmentos (Pires et al. 2002) e estimar a densidade populacional (ex. Mendel \& Vieira 2003).

c) Seleção e uso de habitat: O uso do habitat refere-se ao local do espaço onde o animal posiciona sua área de vida ou realiza suas atividades. De forma geral, os animais não estabelecem sua área de vida aleatoriamente, mas sim em habitats favoráveis. Quando o uso do habitat ocorre em proporções diferentes da disponibilidade dos mesmos no ambiente, diz-se que o animal está selecionando habitat. A definição do habitat de um animal depende de sua biologia e interação com o ambiente, incluindo outros organismos, o que em geral não é óbvio de ser compreendido. $\mathrm{O}$ uso e a seleção do habitat geralmente são avaliados com armadilhas de captura (ex. Ernest \& Mares 1986, Freitas et al. 1997, Dalmagro \& Vieira 2005), mas podem ser estudados com métodos de rastreamento (ex. Moura et al. 2005). Seleção e uso de habitat são atributos individuais, mas podem ser definidos também para uma população ou espécie caso haja um padrão comum à maioria dos indivíduos.

d) Estratificação vertical: Refere-se à organização espacial da comunidade ao longo dos estratos da vegetação (solo, sub-bosque, dossel). Neste caso, o foco é entender como as diferentes espécies em uma comunidade compartilham ou segregam a dimensão vertical do espaço, em uma dada localidade (Vieira 2006). Pode ser avaliada com diversos métodos (Prevedello et al. 2008), principalmente com armadilhas de captura (ex. Vieira \& Monteiro-Filho 2003) e rastreamento dos animais (Cunha \& Vieira 2002).

e) Intensidade de uso vertical: Refere-se à freqüência de uso dos diferentes estratos da vegetação por um indivíduo. Difere da estratificação vertical no sentido de avaliar diferenças intra-específicas no uso do espaço vertical. Para avaliá-la é necessário acompanhar em detalhes a movimentação dos indivíduos, o que pode ser feito, por exemplo, através de carretéis de rastreamento (Cunha \& Vieira 2005, Loretto \& Vieira 2008).

f) Uso de abrigos: Refere-se à seleção de locais para descanso, construção de ninhos ou simplesmente refúgio temporário. Pode ser estudado com o rastreamento dos animais (Miles et al. 1981, Moraes Júnior \& Chiarello 2006) ou com o uso de ninhos artificiais (Loretto 2005).

g) Padrão de agregação espacial: Se refere ao espaçamento dos indivíduos dentro de uma população. O padrão de distribuição espacial pode ser aleatório, homogêneo ou agregado, refletindo a distribuição de recursos e interações intra-específicas (Fernandez et al. 1997). É tradicionalmente avaliado através de armadilhas de captura.

\section{MÉTODOS DE AMOSTRAGEM DE USO DO ESPAÇO}

Diversos métodos foram propostos para o estudo do uso do espaço por mamíferos, todos baseados em um mesmo princípio: identificar os indivíduos e acompanhar seus deslocamentos. O método mais intuitivo e direto é a observação e acompanhamento visual que, salvo raras exceções (ex. CharlesDominique et al. 1983, Ernest \& Mares 1986), é de difícil aplicação aos pequenos mamíferos brasileiros devido ao pequeno porte e habitos crípticos da maioria das espécies (Fonseca et al. 1996). O método de captura-marcação-recaptura (CMR) através de armadilhas que utilizam isca é amplamente utilizado em estudos com pequenos mamíferos, devido à necessidade de capturar os animais. Este método é aplicável ao estudo de vários atributos ecológicos (área de vida, seleção de habitat, estratificação vertical, uso vertical). Recentemente, a técnica tem recebido críticas devido à atratividade das iscas e por não amostrar grande parte da movimentação dos animais (Cunha \& Vieira 2002). A área amostrada pelas armadilhas é geralmente insuficiente para avaliar a área de vida dos animais (Mendel \& Vieira 2003). Este método também não permite observar o uso de refúgios.

Novos métodos foram propostos visando superar as limitações da CMR, dentre os quais se destacam os carretéis de rastreamento (Cunha \& Vieira 2002), a rádio-telemetria (Moraes Júnior \& Chiarello 2005) 
e os ninhos artificiais (Tubelis 2000). Tais métodos permitem descrever a atividade e o uso de refúgios sem influenciar no comportamento dos animais. Delciellos et al. (2006) detalham esses métodos e suas aplicações no estudo de uso do espaço.

\section{LEVANTAMENTO BIBLIOGRÁFICO}

Para as análises da literatura foram considerados apenas estudos que apresentassem resultados inéditos, publicados em periódicos nacionais e internacionais no período de 1945 a 2007. Não foram incluídos nas análises artigos de revisão, capítulos de livros, resumos, monografias, dissertações e teses, além de trabalhos descrevendo técnicas ou métodos. Trabalhos de levantamento (registro de presença-ausência) de espécies não foram considerados. Estudos desenvolvidos em outros países foram consultados para a definição mais geral de conceitos e revisão de técnicas de amostragem.

Em cada estudo foram avaliadas as seguintes variáveis: a) bioma brasileiro estudado (seguindo a classificação de IBGE 2004); b) grupo biológico (Marsupialia ou Rodentia) e gêneros de mamíferos estudados; c) atributo ecológico abordado (área de vida, mobilidade, padrão de agregação espacial, área de vida diária, seleção de habitat, estratificação vertical, intensidade de uso vertical, uso de abrigos); d) técnica de amostragem utilizada (observação direta, armadilhas, rádio-telemetria, carretel de rastreamento, ninhos artificiais).

\section{RESULTADOS \\ BIOMAS ESTUDADOS}

Foram encontrados 58 artigos tratando do uso do espaço por pequenos mamíferos brasileiros. A maior parte destes estudos foi desenvolvida na Mata Atlântica (65\%) e no Cerrado (28\%; Figura 2). Quatro estudos (7\%) foram realizados na Amazônia, apenas um no Pantanal e na Caatinga e nenhum nos Campos Sulinos.

\section{GRUPOS BIOLÓGICOS ESTUDADOS}

O número de estudos foi similar para marsupiais e roedores ( $n=44$ e $n=40$ estudos respectivamente), mas a proporção variou bastante entre biomas (Figura 3). Na Mata Atlântica, os marsupiais e roedores foram analisados em $86 \%$ e $53 \%$ dos trabalhos respectivamente (Figura 3b), mas no Cerrado essas proporções mudam para 44\% e 100\% (Figura 3c). Os quatro estudos realizados na Amazônia relataram resultados para ambos os grupos.

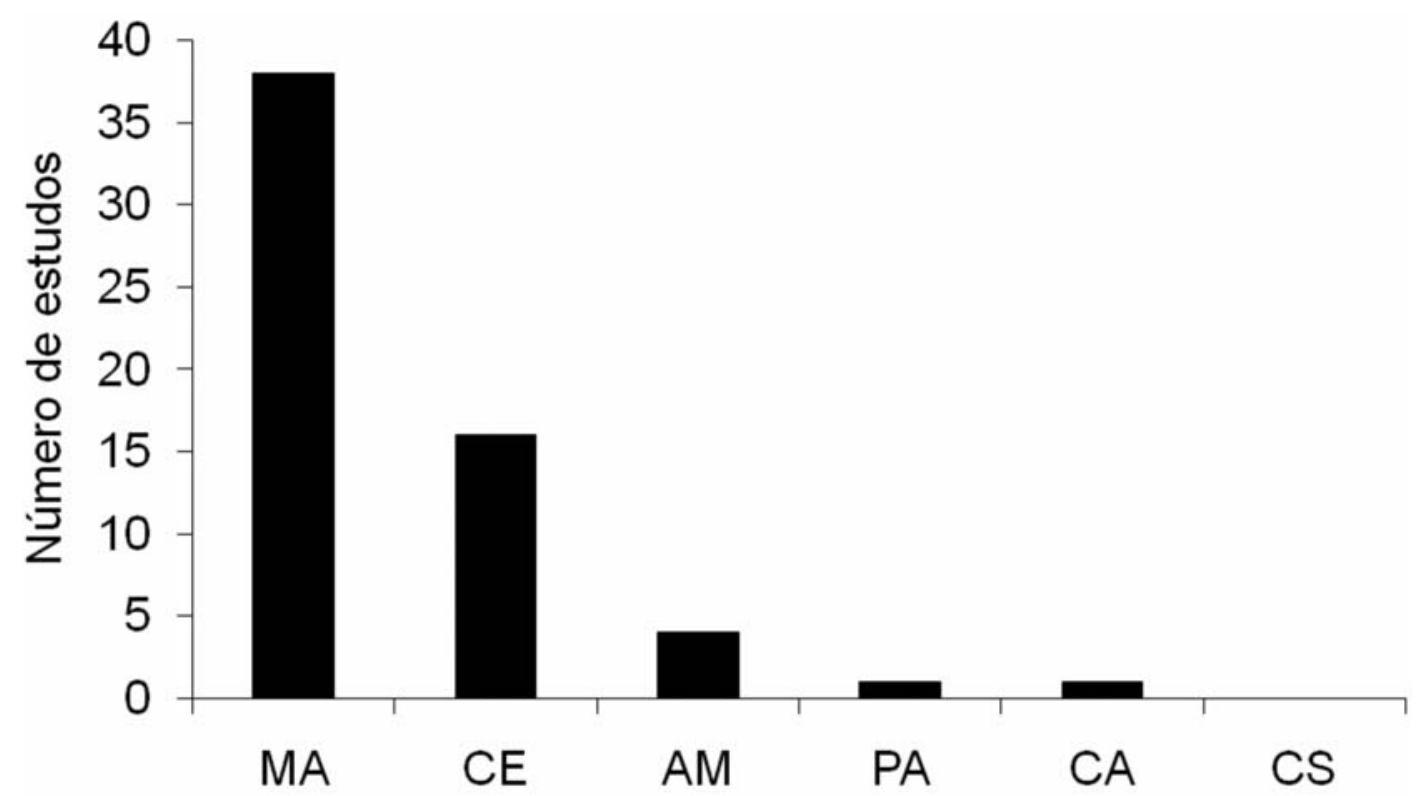

Figura 2. Número de estudos publicados sobre uso do espaço por pequenos mamíferos em cada bioma brasileiro. MA: Mata Atlântica; CE: Cerrado; AM: Amazônia; PA: Pantanal; CS: Campos Sulinos; CA: Caatinga.

Figure 2. Number of publications regarding space use by small mammals in different Brazilian biomes. MA: Atlantic Forest; CE: Cerrado; AM: Amazon; PA: Pantanal; CS: Campos Sulinos; CA: Caatinga. 

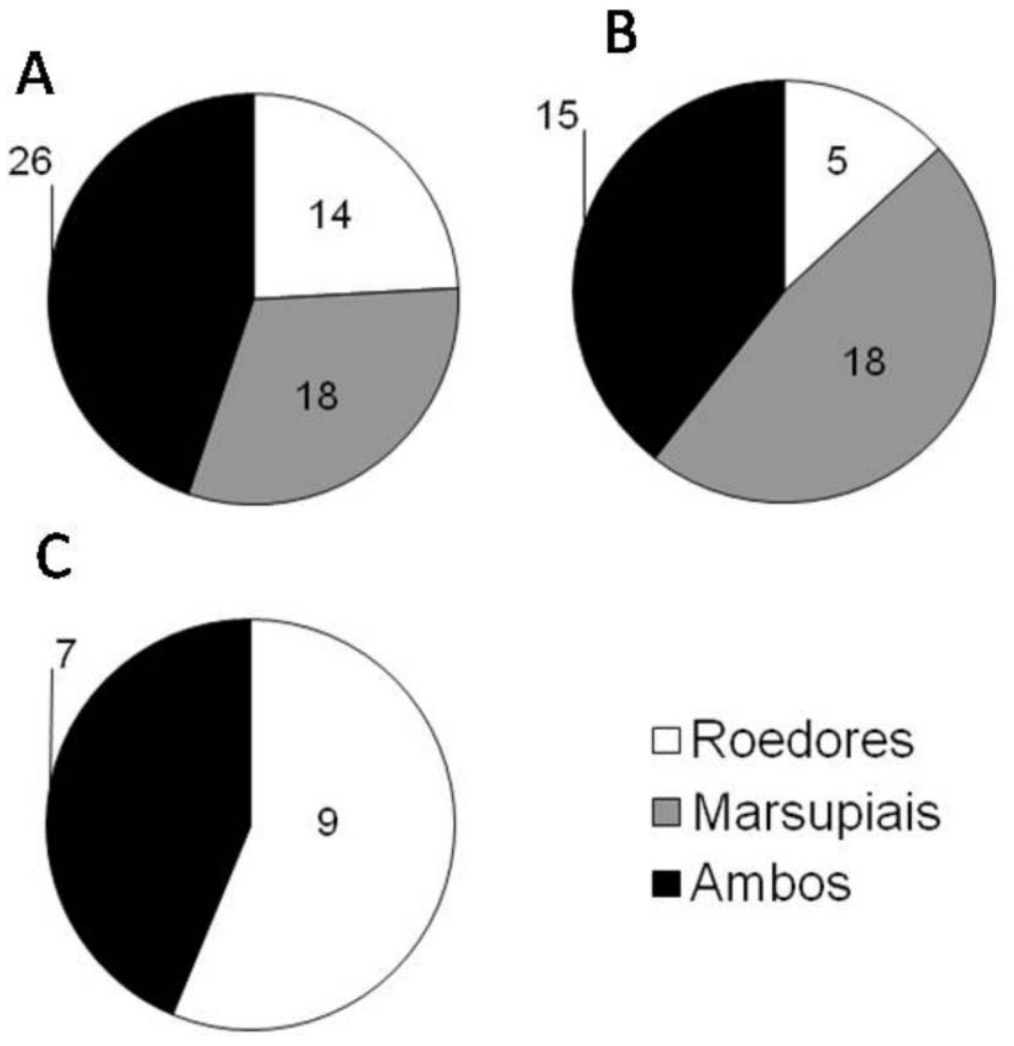

Figura 3. Número de artigos analisando uso do espaço por pequenos mamíferos brasileiros (marsupiais, roedores ou ambos). (A) todos os estudos; (B) estudos realizados na Mata Atlântica; $(\mathrm{C})$ estudos realizados no Cerrado. Os valores indicam o número de artigos publicados por grupo e bioma.

Figure 3. Number of publications on space use by Brazilian small mammals (marsupials, rodents, or both). (A) all studies; (B) studies conducted in the Atlantic Forest; (C) studies conducted in the Cerrado. Values indicate the number of published papers with each animal group and biome.

Para marsupiais houve uma predominância de estudos com os gêneros Didelphis (19), Philander $(\mathrm{n}=13)$, Metachirus $(\mathrm{n}=10)$ e Micoureus $(\mathrm{n}=10)$ (Figura 4a, Tabela II). A maioria dos estudos com esses gêneros foi desenvolvida na Mata Atlântica, sendo que os estudos com Philander ficaram restritos a este bioma. Em relação aos roedores, predominaram estudos com Oligoryzomys ( $\mathrm{n}=17)$, Necromys $(\mathrm{n}=15)$ e Akodon ( $\mathrm{n}=13$ ) (Figura 4b, Tabela II). Apesar de terem sido realizados mais estudos com roedores no Cerrado, os trabalhos desenvolvidos na Mata Atlântica registraram um maior número de gêneros (Figura 4b).

\section{ATRIBUTOS ECOLÓGICOS ABORDADOS}

Os atributos mais estudados foram: seleção/uso de habitat ( $40 \%$ dos estudos), área de vida (24\%) e mobilidade (19\%; Figura 5). O conceito de área de vida diária, relacionado ao uso de carretéis de rastreamento, foi o menos estudado (3\%). Dentre os 23 trabalhos de seleção/uso de habitat, 39\% não avaliaram a disponibilidade de habitat restringindo-se, assim, a descrever seu uso. Dos 14 estudos tratando de área de vida, 11 foram desenvolvidos na Mata Atlântica e apenas três foram realizados no Cerrado. Apenas 14\% dos estudos avaliaram de alguma forma o uso vertical do espaço, seja analisando a intensidade de uso vertical (5\%) ou a estratificação vertical (9\%).

\section{TÉCNICAS DE AMOSTRAGEM UTILIZADAS}

A técnica mais usada foi a de captura-marcaçãorecaptura com armadilhas ( $75 \%$ dos estudos), seguida do carretel de rastreamento (17\%) e rádio-telemetria (7\%; Figura 6). Apenas dois trabalhos (Alho \& Villela 1984, Ernest \& Mares 1986) realizaram observação direta e um trabalho utilizou ninhos artificiais (Monteiro-Filho \& Marcondes-Machado 1996).

\section{DISCUSSÃO}

\section{BIOMAS ESTUDADOS}

O conhecimento atual sobre o uso do espaço no Brasil é proveniente principalmente de estudos desenvolvidos na Mata Atlântica e no Cerrado. Tal 


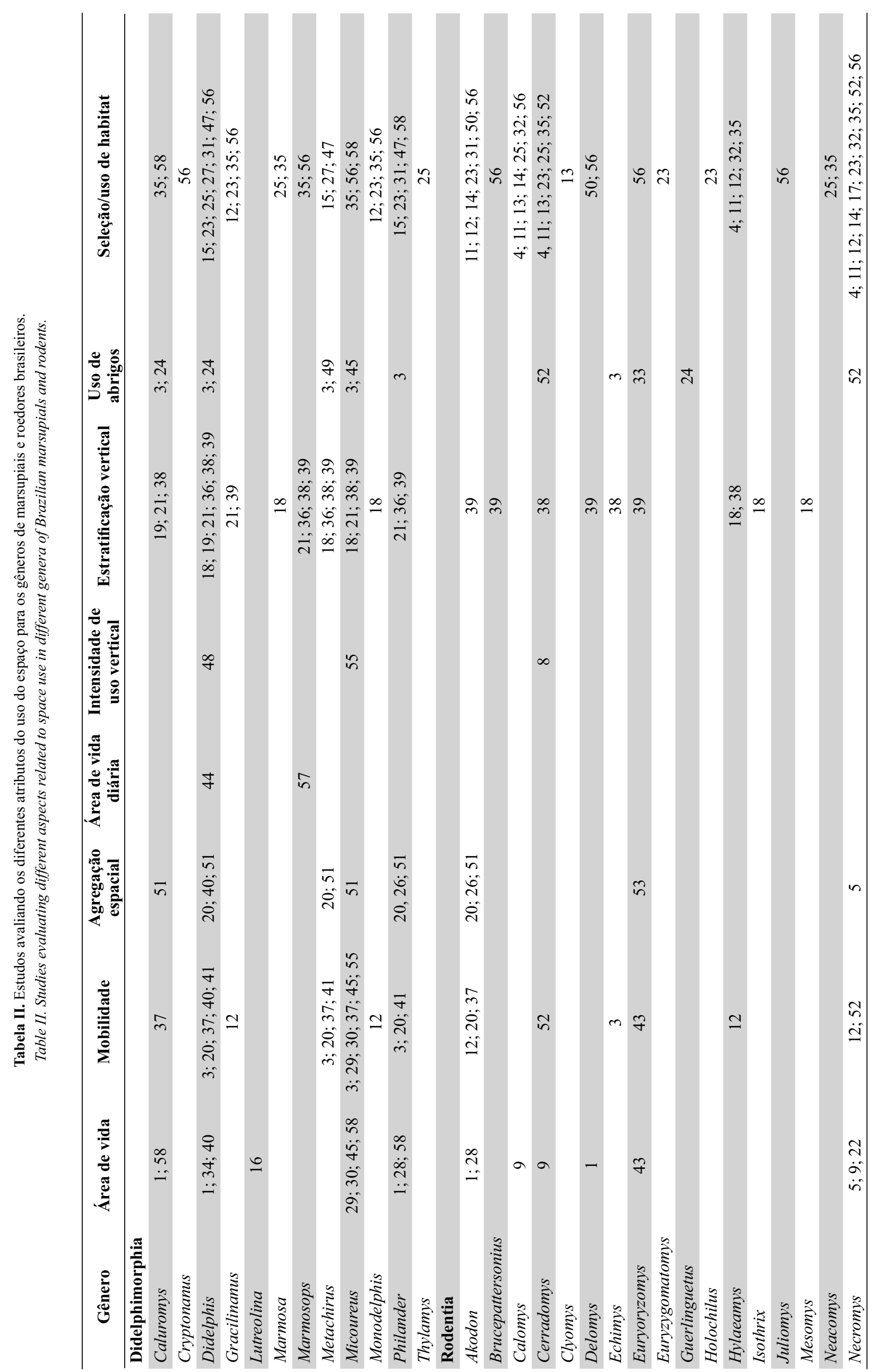




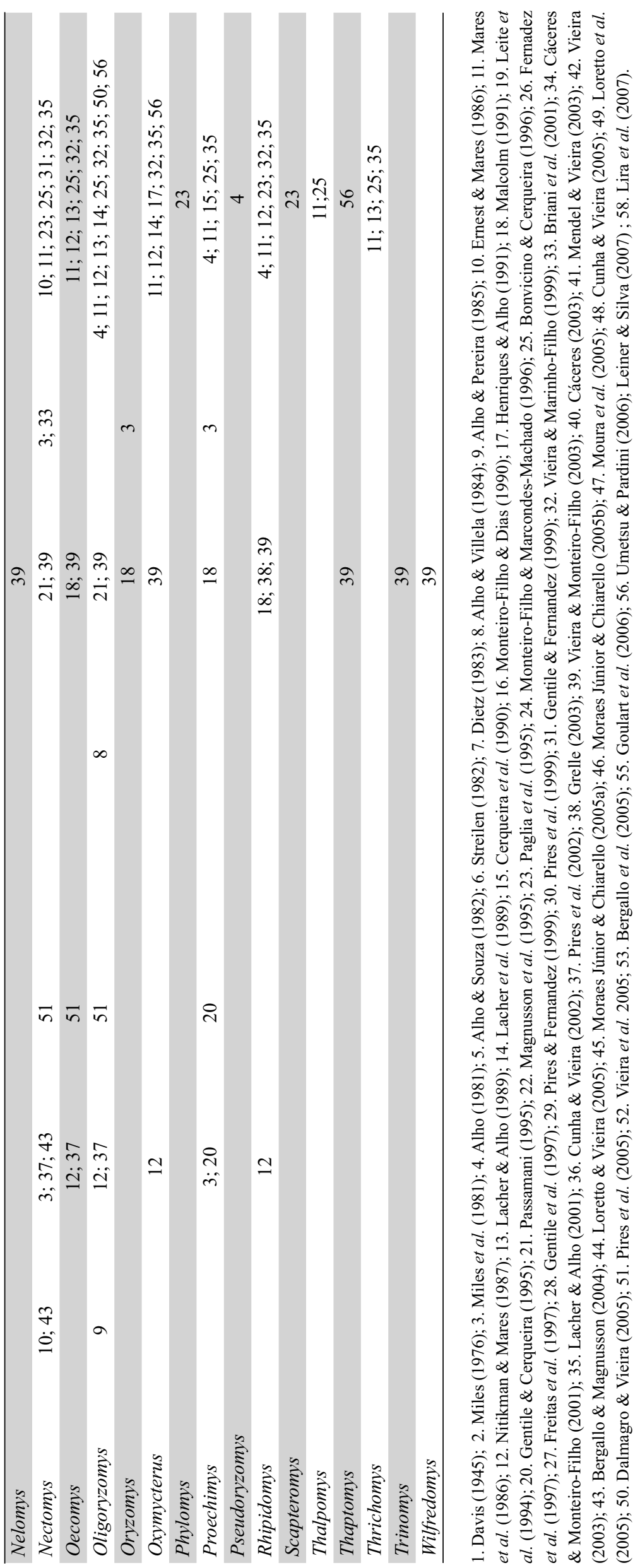




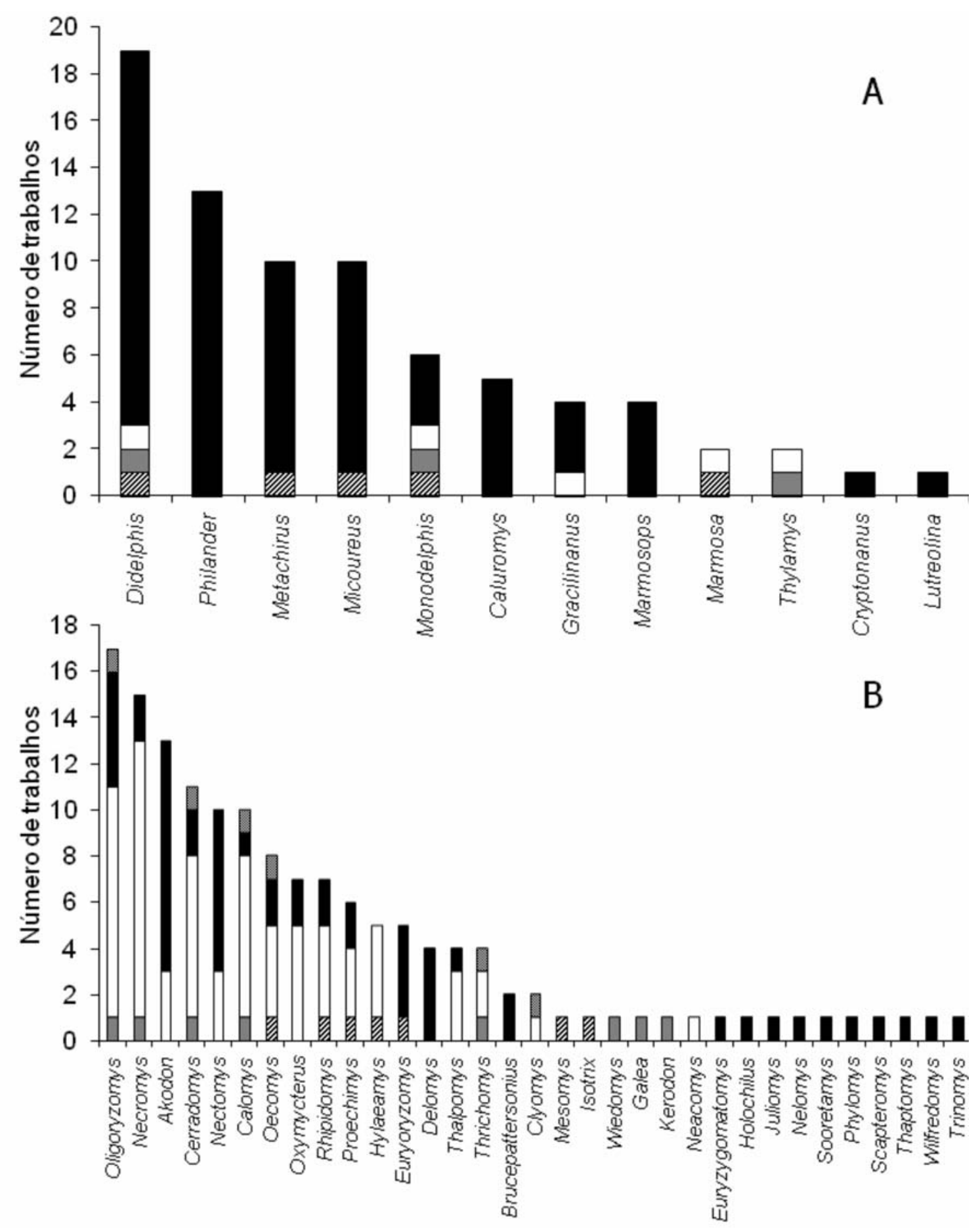

घamazônia $\square$ Caatinga $\square$ Cerrado $\square$ MataAtlântica $\square$ Pantanal

Figura 4. Número de estudos de uso do espaço realizados com cada gênero de marsupiais (A) e roedores (B) nos diferentes biomas brasileiros.

Figure 4. Number of space use studies conducted with different genera of (A) marsupials and (B) rodents in the different Brazilian biomes.

padrão se deve provavelmente à proximidade aos grandes centros urbanos e à presença de grupos de pesquisa estabelecidos nesses centros, ligados principalmente a universidades com tradição em pesquisa. Nos demais biomas brasileiros existem poucos pesquisadores voltados ao estudo de pequenos mamíferos. A escassez de estudos na Amazônia é especialmente relevante, dada a alta riqueza de pequenos mamíferos no bioma (Emmons 1997). Na Caatinga e no Pantanal a ausência de estudos é mais agravante em termos teóricos, visto que suas fitofisionomias, recursos e condições são bastante peculiares, podendo gerar novos padrões de uso do espaço por pequenos mamíferos. A distribuição dos 


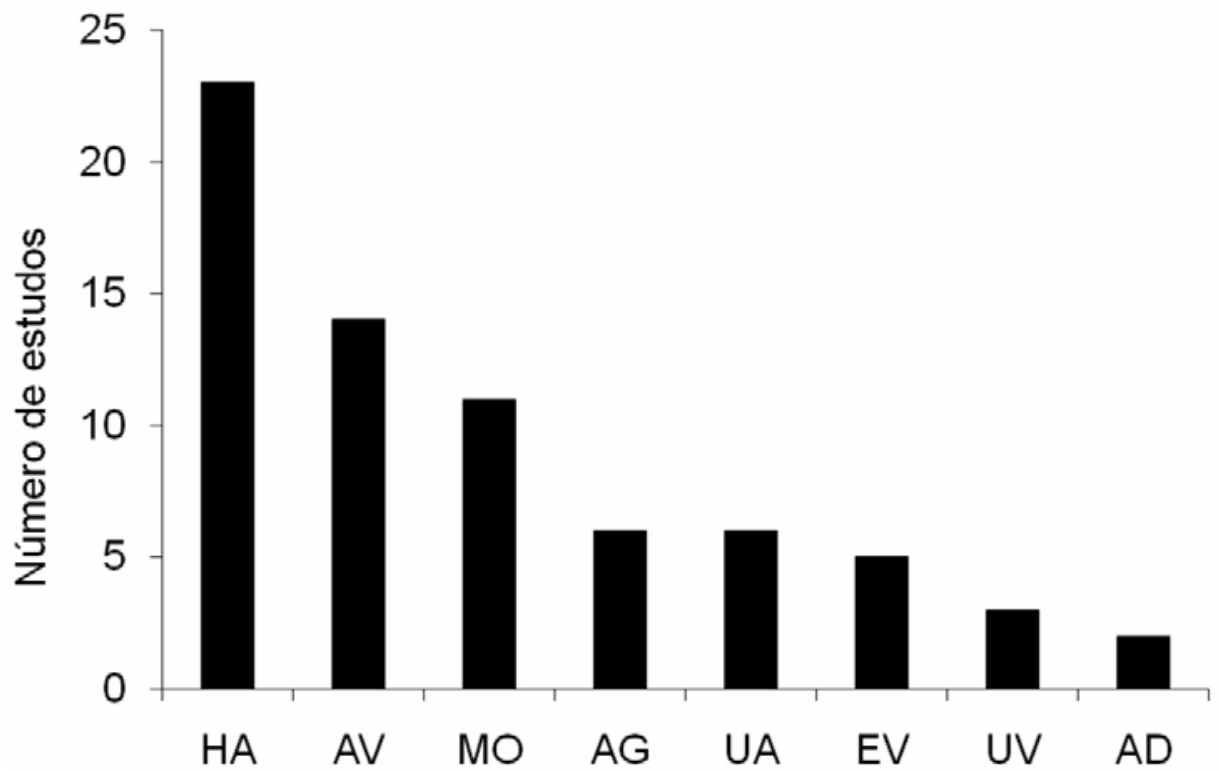

Figura 5. Atributos abordados nos estudos sobre uso do espaço por pequenos mamíferos brasileiros. HA: seleção/uso de habitat; AV: área de vida; MO: mobilidade; AG: padrão de agregação espacial; UA: uso de abrigos; EV: estratificação vertical; UV: intensidade de uso vertical; AD: área de vida diária. Figure 5. Different traits analyzed in studies of space use by Brazilian small mammals. HA: habitat utilization/selection; AV: home range; MO: mobility; AG: spatial distribution of population aggregates; UA: nest use; EV: vertical stratification; UV: intensity of vertical use; AD: daily home range.

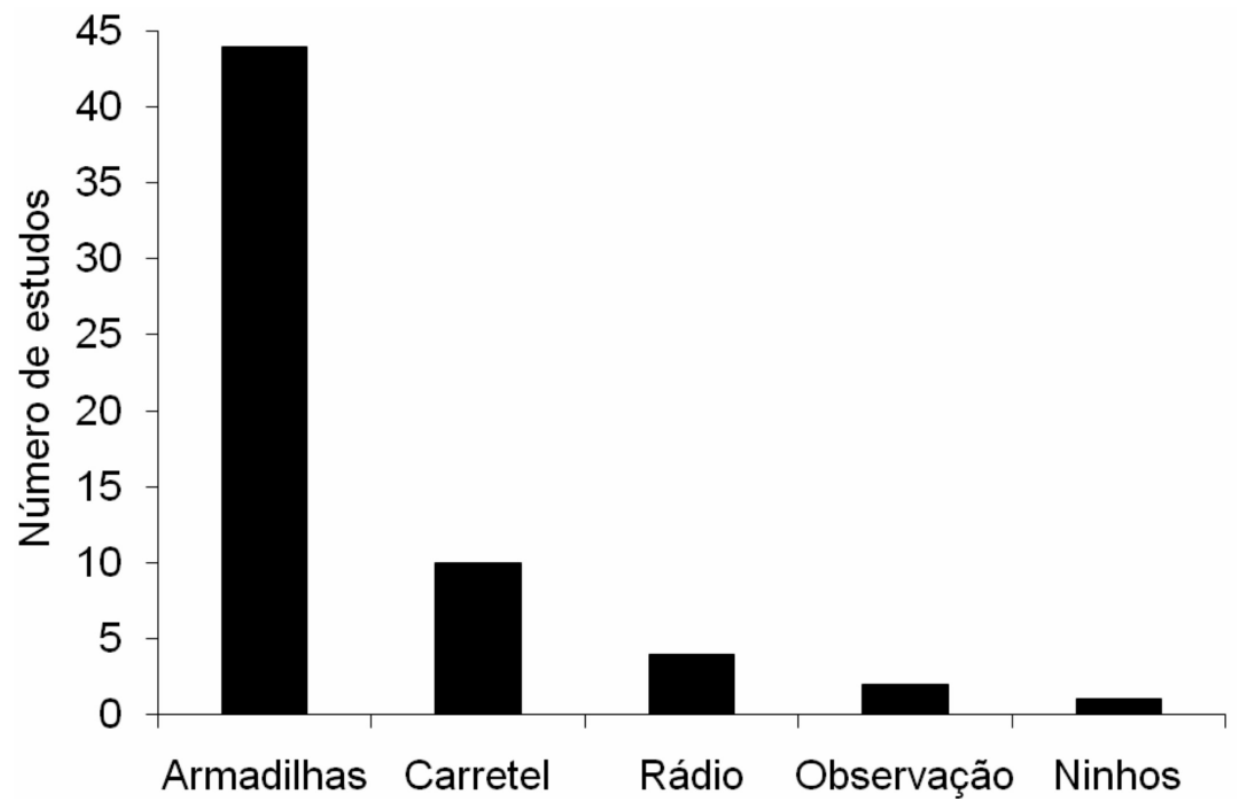

Figura 6. Métodos de amostragem utilizados para avaliar o uso do espaço por pequenos mamíferos brasileiros.

Figure 6. Sampling methods used to evaluate space use by Brazilian small mammals.

estudos sobre o uso do espaço deve estar representando a distribuição do conhecimento ecológico geral sobre os pequenos mamíferos brasileiros.

\section{GRUPOS BIOLÓGICOS ESTUDADOS}

A predominância de estudos com roedores no Cerrado e com marsupiais na Mata Atlântica sugere que a escolha das espécies estudadas dependeu da abundância e capturabilidade das mesmas. Embora a riqueza de espécies seja semelhante entre os dois biomas (ex. Bonvicino et al. 2002), estudos em Cerrado tendem a capturar mais roedores (ex. Mares et al. 1986), enquanto estudos na Mata Atlântica tendem a capturar mais marsupiais (ex. Macedo et al. 2007). No Cerrado, as espécies mais abundantes geralmente são roedores (ex. Marinho-Filho et al. 2002), enquanto na Mata Atlântica as espécies de marsupiais são geralmente as mais abundantes (ex. Prevedello et al. 2008). O sucesso de captura 
influencia diretamente o número amostral obtido pelos pesquisadores, que concentram então suas análises nas espécies mais comuns. Isso é demonstrado pela concentração dos estudos nos gêneros Didelphis, Philander, Oligoryzomys e Necromys (Figura 4, Tabela II), os quais são geralmente os mais comuns nas comunidades estudadas (Marinho-Filho et al. 2002, Macedo et al. 2007). Esse padrão de escolha dos grupos limita o conhecimento e pode causar desvios inesperados nos padrões gerados, exigindo assim estudos de longo prazo e com maior esforço amostral (ex. Prevedello et al. 2008).

\section{ATRIBUTOS ECOLÓGICOS ABORDADOS}

Os dois conceitos mais estudados, seleção/uso do habitat e área de vida, são antigos e têm longa tradição na Ecologia Animal (Lima \& Zollner 1996, Garshelis 2000). Por outro lado, a estratificação vertical, intensidade de uso vertical e área de vida diária foram pouco estudados por serem conceitos recentes. Até o início da década de 1990,80\% dos estudos avaliaram área de vida ou seleção de habitat, enquanto apenas um (Alho \& Villela 1984) avaliou intensidade de uso vertical e nenhum avaliou estratificação vertical ou área de vida diária. Os estudos sobre a estratificação vertical no Brasil começaram com o estudo pioneiro de Malcolm (1991), que mostrou que diversas espécies são capturadas apenas ou principalmente nos estratos superiores da mata, o que gerou uma nova abordagem no estudo de pequenos mamíferos no Brasil. Os estudos de área de vida diária são uma abordagem ainda mais recente, ligados à utilização de carretéis de rastreamento (Loretto \& Vieira 2005).

É possível notar diferenças de enfoque entre os biomas nos atributos ecológicos estudados. O uso/ seleção de habitat, por exemplo, recebeu atenção principalmente no Cerrado. Cinqüenta por cento dos estudos sobre seleção de habitat foram conduzidos no Cerrado, enquanto apenas $26 \%$ dos estudos em geral foram desenvolvidos no bioma. Isso se deveà conspícua heterogeneidade estrutural do Cerrado, que apresenta um mosaico de fitofisionomias variando de formações abertas e de savana até formações florestais (Rizzini 1979, Oliveira-Filho \& Ratter 2002). Em relação à área de vida, apenas três estudos foram realizados no Cerrado, o que reflete um enfoque maior no uso/ seleção do habitat nas diferentes fitofisionomias do bioma, demandando novos estudos sobre o tema. Por outro lado, os estudos de estratificação vertical ficaram restritos aos biomas Mata Atlântica e Amazônia, em função da maior complexidade daqueles biomas. Seria interessante estudar a estratificação vertical nos ambientes florestados do Cerrado, comparando a estrutura das comunidades com as da Amazônia e Mata Atlântica.

Pouquíssimos estudos avaliaram de alguma forma o uso vertical do espaço. Isso se deve em parte às dificuldades de acesso aos estratos superiores da mata, limitação superada recentemente com o uso de plataformas suspensas (Malcolm 1991, Vieira \& Monteiro-Filho 2003), carretéis de rastreamento (Cunha \& Vieira 2002) e ninhos artificiais (Loretto 2005, Prevedello et al. 2008). Essa limitação é especialmente relevante no caso dos estudos conduzidos na Mata Atlântica e Amazônia, dada a marcada estratificação vegetacional nesses biomas. Visto que a maioria dos marsupiais brasileiros e várias espécies de roedores são arborícolas ou escansoriais (Fonseca et al. 1996), é fundamental que novos estudos avaliem o espaço de forma tridimensional (ex. Meserve 1977, Grelle 1996).

\section{TÉCNICAS UTILIZADAS}

A técnica de CMR utilizando armadilhas foi a principal ferramenta para o estudo do uso do espaço, apesar de suas limitações. A maioria dos estudos populacionais de pequenos mamíferos utiliza CMR em armadilhas dispostas em grades, gerando dados que são utilizados secundariamente para avaliar o uso do espaço. Apesar de ser aplicável ao estudo de vários atributos do uso do espaço, o método tem recebido críticas devido à atratividade das iscas e por não amostrar grande parte da movimentação dos animais (Cunha \& Vieira 2002). Além disso, como o delineamento dos estudos de CMR visa estimar parâmetros populacionais, a avaliação do uso do espaço fica limitada pelo tamanho em geral insuficiente das grades de armadilha ou transeções. Outra desvantagem do método é não avaliar o uso de abrigos e ninhos. Apesar dessas limitações, a técnica continuarásendoamplamenteutilizada, eventualmente em conjunto com métodos mais modernos.

A técnica do carretel de rastreamento foi a segunda mais utilizada com $82 \%$ dos estudos realizados nos 
últimos sete anos (revisão em Delciellos et al. 2006). Este método traz diversas vantagens ao estudo ecológico de pequenos mamíferos, em especial em relação ao uso de abrigos (ex. Briani et al. 2003), seleção de habitat (Moura et al. 2005) e estratificação vertical das espécies (Cunha \& Vieira 2002). A técnica permite alta resolução espacial e detalhamento dos movimentos, mas tem a desvantagem de amostrar o uso do espaço somente em curtas escalas espaço-temporais. A rádio-telemetria foi também utilizada apenas recentemente e em somente quatro estudos, apesar de permitir o estudo detalhado de quase todos os atributos de uso do espaço (Jacob \& Rudran 2003). O alto custo e o grande esforço de campo parecem ser suas principais desvantagens. A técnica de ninhos artificiais, que potencialmente oferece vantagens ao estudo comportamental e demográfico de mamíferos arborícolas (Loretto 2005), teve apenas um estudo publicado até 2007 (Monteiro-Filho \& MarcondesMachado 1996). Por fim, a observação direta é pouco adequada ao estudo do uso do espaço por pequenos mamíferos, devido aos hábitos predominantemente crípticos dos animais.

\section{CONSIDERAÇÕES FINAIS}

O conhecimento atual sobre o uso do espaço por pequenos mamíferos brasileiros é limitado devido à amostragem assimétrica dos biomas e grupos estudados, além dos atributos abordados e técnicas utilizadas. Quatro dos seis biomas brasileiros (Amazônia, Caatinga, Campos Sulinos e Pantanal) apresentam grande escassez de informações. Novas técnicas, diferentes das tradicionais armadilhas de captura, devem ser utilizadas como ferramentas em estudos delineados especificamente para avaliar o uso do espaço. Especial atenção deve ser dada ao uso vertical do espaço em biomas florestais como a Mata Atlântica e a Amazônia e em formações florestais do Cerrado e Caatinga.

Acreditamos que o Brasil pode contribuir para importantes avanços teóricos na área. A alta riqueza de espécies, aliada à grande variedade de ecossistemas que possui, coloca o país como potencial gerador de novos modelos e teorias relacionadas ao tema. Além disso, a alta complexidade estrutural das florestas brasileiras pode trazer novas abordagens ao entendimento tridimensional da ecologia de mamíferos tropicais.
AGRADECIMENTOS: À Paula Ferreira (UFRJ) e dois revisores anônimos pelas críticas e sugestões ao manuscrito. Aos colegas do Laboratório de Vertebrados/ UFRJ pelas diversas discussões que enriqueceram o estudo. O Conselho Nacional de Desenvolvimento Científico e Tecnológico (CNPq) e a Coordenação de Aperfeiçoamento de Pessoal de Nível Superior (CAPES) forneceram financiamento com bolsas de mestrado e doutorado.

\section{REFERÊNCIAS}

ALHO, C.J.R. 1981. Small mammal populations of Brazilian Cerrado: The dependence of abundance and diversity on habitat complexity. Revista Brasileira de Biologia, 41(1): 223-230.

ALHO, C.J.R. \& PEREIRA, L.A. 1985. Population ecology of a Cerrado rodent community in Central Brazil. Revista Brasileira de Biologia, 45: 597-607.

ALHO, C.J.R.; PEREIRA, L.A. \& PAULA, A.C. 1986. Patterns of habitat utilization by small mammal populations in cerrado biome of Central Brazil. Mammalia, 50: 447-460.

ALHO, C.J.R. \& SOUZA, M.J. 1982. Home range and use of space in Zygodontomys lasiurus (Cricetidae, Rodentia) in the Cerrado of central Brazil. Annals of Carnegie Museum, 51: 127-132.

ALHO, C.J.R. \& VILLELA, O.M.M. 1984. Scansorial Ability in Oryzomys eliurus and O. subflavus (Rodentia: Cricetidae) from the Cerrado. Revista Brasileira de Biologia, 44: 403-408.

August, P.V. 1983. The role of habitat complexity and heterogeneity in structuring tropical mammal communities. Ecology, 64: 1495-1507.

BERGALLO, H.G. 1990. Fatores determinantes do tamanho da área de vida em mamíferos. Ciência e Cultura, 42(12): 1067 1072.

BERGALLO, H.G.; LUZ, J.L.; RAÍCES, D.S.L.; HATANO, F. \& HATANO, F.M. 2005. Habitat use by Oryzomys subflavus (Rodentia) in an open shrubland formation in Restinga de Jurubatiba National Park, RJ, Brazil. Brazilian Journal of Biology, 65(4): 538-588.

BERGALLO, H.G. \& MAGNUSSON, W.E. 2004. Factors affecting the use of space by two rodent species in Brazilian Atlantic forest. Mammalia 68(2-3): 121-132.

BONVICINO, C.R. \& CERQUEIRA, R. 1996. Habitat use by small mammals of upper Araguaya River. Revista Brasileira de Biologia, 56: 761-767.

BONVICINO, C.R.; LINDBERGH, S.M. \& MAROJA, L.S. 2002. Small non-flying mammal from conserved and altered areas of Atlantic Forest and Cerrado: comments on their potential use for monitoring environment. Brazilian Journal of Biology, 62(4B): 765-774. 
BRIANI, D.C.; VIEIRA, E.M. \& VIEIRA, M.V. 2001. Nests and nesting sites of Brazilian forest rodents (Nectomys squamipes and Oryzomys intermedius) as revealed by a spool-and-line device. Acta Theriologica, 46: 331-334.

BURT, W.H. 1943. Territoriality and home range concepts as applied to mammals. Journal of Mammalogy, 24(3): 346-352.

CÁCERES, N.C \& MONTEIRO-FILHO, E.L.A. 2001. Food habits, home range and activity of Didelphis aurita (Mammalia, Marsupialia) in a forest fragment of southern Brazil. Studies on Neotropical Fauna and Environment, 36: 85-92.

CÁCERES, N.C. 2003. Use of the space by the opossum Didelphis aurita in a forest fragment of southern Brazil. Revista Brasileira de Zoologia, 20(2): 315-322.

CÁCERES, N.C. \& MONTEIRO-FILHO, E.L.A. 2006. Área de vida de marsupiais brasileiros. Pp 203-215. In: N.C. Cáceres \& E.L.A Monteiro Filho (eds). Os marsupiais do Brasil: biologia, ecologia e evolução. Editora da UFMS, Campo Grande. 364p.

CERQUEIRA, R.; FERNANDEZ, F. \& NUNES, M.F.Q.S. 1990. Mamíferos da Restinga de Barra de Marica. Papeis Avulsos de Zoologia, 37: 141-157.

CHARLES-DOMINIQUE, P. 1983. Ecology and social adaptations in didelphid marsupials: comparison with eutherians of similar ecology. Pp 395-422. In: J.F. Eisenberg \& D.G. Kleiman (eds). Advances in the study of mammalian behavior. American Society of Mammalogy Special Publication, Pennsylvania. 753p.

CUNHA, A.A. \& VIEIRA, M.V. 2002. Support diameter, incline, and vertical movements of four didelphid marsupials in the Atlantic Forest of Brazil. Journal of Zoology, 258: 419-426.

CUNHA, A.A. \& VIEIRA, M.V. 2004. Two bodies cannot occupy the same place at the same time, or the importance of space in the ecological niche. Bulletin of the Ecological Society of America, 85: 25-26.

CUNHA, A.A. \& VIEIRA, M.V. 2005. Age, season, and vertical use of the Atlantic rainforest by the common opossum, Didelphis aurita Wied 1826. Acta Theriologica, 50: 551-560.

DALMAGRO, A. \& VIEIRA, E.M. 2005. Patterns of habitat utilization of small rodents in an area of Araucaria forest in Southern Brazil. Austral Ecology, 30: 353-362.

DAVIS, D.E. 1945. The home range of some Brazilian mammals. Journal of Mammalogy, 26(2): 119-127.

DELANY, M.J. 1974. The ecology of small mammals. The Camelot Press Ltd, Southampton.

DELCIEllos, A.C.; LORETTO, D. \& VIEIRA, M.V. 2006. Novos métodos no estudo da estratificação vertical de marsupiais neotropicais. Oecologia Brasiliensis, 10: 135-156.
EMMONS, L.H. 1997. Neotropical rainforest mammals: a field guide. (Second edition). The University of Chicago Press, Chicago. 307p.

DIETZ, J.M. 1983. Notes on the natural history of some small mammals in Central Brazil. Journal of Mammalogy, 64(3): 521-523.

ERNEST, K.A. \& MARES, M.A. 1986. Ecology of Nectomys squamipes, the neotropical water rat, in central Brazil: Home range, habitat selection, reproduction and behavior. Journal of Zoology, 210(4): 599-612.

FERNANDEZ, F.A.S.; FREITAS, S.R. \& CERQUEIRA, R. 1997. Density dependence in within-habitat spatial distribution: Contrasting patterns for a rodent and a marsupial in southeastern Brazil. Ciência e Cultura, 49 (1/2): 127-129.

FREITAS, S.R.; ASTÚA DE MORAES, D.; SANTORI, R.T. \& CERQUEIRA, R. 1997. Habitat preference and food use by Metachirus nudicaudatus and Didelphis aurita in a restinga forest at Rio de Janeiro. Revista Brasileira de Biologia, 57: 93-98.

FONSECA, G.A.B.; HERMANN, G.; LEITE, Y.L.R.; MITTERMEIER, R.A,; RYLANDS, A.B. \& PATTON, J.L. 1996. Lista anotada dos mamíferos do Brasil. Occasional papers in Conservation Biology, 4: 1-38.

GARSHELIS, D.L. 2000. Delusions in habitat evaluation: measuring use, selection, and importance. Pp 111-164 In: L. Boitani \& T.K. Fuller (eds). Research techniques in animal ecology: controversies and consequences. Columbia University Press, New York. 442p.

GENTILE, R. \& CERQUEIRA, R. 1995. Movement patterns of five species of small mammals in a Brazilian Restinga. Journal of Tropical Ecology, 11: 671-677.

GENTILE, R.; D’ANDREA, P.S. \& CERQUEIRA, R. 1997. Home ranges of Philander frenata and Akodon cursor in a Brazilian Restinga (coastal shrub land). Mastozoologia Neotropical, (2): 105-112.

GENTILE, R. \& FERNANDEZ, F.A.S. 1999. Influence of habitat structure on a streamside small mammal community in a Brazilian rural area. Mammalia, 63(1): 29-10.

GOULART, F.V.B.; SOUZA, F.L.; PAVESE, H.B. \& GRAIPEL, M.E. 2006. Estrutura populacional e uso do estrato vertical por Micoureus paraguayanus (Didelphimorphia, Didelphidae) em fragmentos de Floresta Atlântica de planície no sul do Brasil. Biotemas, 19 (3): 45-53.

GRELLE, C.E.V. 1996. How to estimate three-dimensional home range? A proposal from a study with Atlantic Forest small mammals. What's Up? The Newsletter of International Canopy Network, 2(2): 3-3.

GRELLE, C.E.V. 2003. Forest structure and vertical 
stratification of small mammals in a secondary Atlantic Forest, Southeastern Brazil. Studies on Neotropical Fauna and Environment, 35: 1-9.

HENRIQUES, R.P.B. \& ALHO, C.J.R. 1991. Microhabitat selection by two rodent species in the Cerrado of Central Brazil. Mammalia, 55: 49-56.

INSTITUTO BRASILEIRO DE GEOGRAFIA E ESTATÍSTICA. 2004. Mapa de biomas do Brasil: Primeira aproximação. $<\mathrm{ftp}$ :/ftp.ibge.gov.br/Cartas_e_Mapas/Mapas_Murais/>. Acesso em 30/06/2008.

JACOB, A.A. \& RUDRAN, R. 2003. Radiotelemetria em estudos populacionais. Pp 285-341. In: L. Cullen Jr., R. Rudran \& C. Valladares-Padua (eds). Métodos de Estudos em Biologia da Conservação e Manejo da Vida Silvestre. Editora da UFPR \& Fundação O Boticário de Proteção à Natureza. Curitiba. 667p.

KOEPPL, J.M.; SLADE, N.A.; HARRIS, K.S. \& HOFFMANN, R.S. 1977. A three-dimensional home range model. Journal of Mammalogy, 58: 213-220.

LACHER Jr, T.E. \& ALHO, C.J.R. 1989. Microhabitat Use among Small Mammals in the Brazilian Pantanal. Journal of Mammalogy, 70(2): 396-401.

LACHER Jr, T.E.; MARES, M.A. \& ALHO, C.J.R. 1989. The Structure of a Small Mammal Community in a Central Brazilian Savanna. Pp 137-162. In: K.H. Redford; J.F. Eisenberg. (eds.). Advances in Neotropical Mammalogy. Sandhill Crane Press, Florida. 614p.

LACHER Jr, T.E. \& ALHO, C.J.R. 2001. Terrestrial small mammals richness and habitat associations in an Amazon forest-cerrado contact zone. Biotropica, 33(1): 171-181.

LEINER, N.O. \& SILVA, W.R. 2007. Effects of resource availability on the use of space by the mouse opossum Marmosops paulensis (Didelphidae) in a montane Atlantic forest area in southeastern Brazil. Acta Theriologica, 52(2): 197-204.

LEITE, Y.L.; STALLINGS, J. \& COSTA, L.P. 1994. Partição de recursos entre espécies simpátricas de marsupiais na Reserva Biológica de Poço das Antas, RJ. Revista Brasileira de Biologia, 54: 525-536.

LIMA, S.L. \& ZOLLNER, P.A. 1996. Towards a behavioral ecology of ecological landscapes. Trends in Ecology \& Evolution, 11(3): 131-135.

LIRA, P.K.; FERNANDEZ, F.A.S.; CARLOS, H.S.A. \& CURZIO, P.L. 2007. Use of a fragmented landscape by three species of opossum in southeastern Brazil. Journal of Tropical Ecology, 23: 427-435.

LORETTO, D.; RAMALHO, E.; VIEIRA, M.V. 2005. Defense behavior and nest arquitecture of Metachirus nudicaudatus Desmarest, 1817 (Marsupialia; Didelphidae). Mammalia, 69: 417-419.
LORETTO, D. \& VIEIRA, M.V. 2005. The effects of reproductive and climatic seasons on movements in the black-eared opossum (Didelphis aurita Wied-Neuwied, 1826). Journal of Mammalogy, 86(2): 287-293.

LORETTO, D. \& VIEIRA, M.V. no prelo. Use of space by the marsupial Marmosops incanus (Didelphimorphia, Didelphidae) in the Atlantic Forest, Brazil. Mammalian Biology, 73: 255-261.

MACEDO, J.; LORETTO, D.; MELLO, M.C.S.; FREITAS, S.; VIEIRA, M.V. \& CERQUEIRA, R. 2007. História natural dos mamíferos de uma área perturbada do Parque Nacional da Serra dos Órgãos, Rio de Janeiro, Brasil. Pp 165-181. In: C. Cronemberguer \& E.V. Castro (eds.) Ciência e Conservação na Serra dos Órgãos. IBAMA, Brasilia. 296p.

MAGNUSSON, W.E.; FRANCISCO, A.L. \& SANAIOTTI, T.M. 1995. Home range size and territoriality in Bolomys lasiurus (Rodentia: Muridae) in an Amazonian savanna. Journal of Tropical Ecology, 11: 179-188.

MALCOLM, J.R. 1991. Comparative abundances of Neotropical small mammals by trap height. Journal of Mammalogy, 72: 188-192.

MARES M.A.; ERNEST, K.A. \& GETTINGER, D.D. 1986. Small mammal community structure and composition in the Cerrado province of central Brazil. Journal Tropical Ecology, 2: $289-300$

MARINHO FILHO, J.; RODRIGUES, F.H.G.; JUAREZ, K.M. 2002. The Cerrado mammals: diversity, ecology and natural history. Pp 266-284. In: P.S. Oliveira \& R.J. Marquis (eds). The Cerrados of Brazil: Ecology and Natural History of a Neotropical Savanna. Columbia University Press. New York. $424 \mathrm{p}$.

MENDEL, S.M. \& VIEIRA, M.V. 2003. Movement distances and density estimation of small mammals using the spooland-line technique. Acta Theriologica, 48: 289-300.

MESERVE, P.L. 1977. Three-dimensional home ranges of cricetid rodents. Journal of Mammalogy, 58: 549-558.

MILES, M.A. 1976. A simple method of tracking mammals and locating Triatomine vectors of Trypanosoma cruzi in Amazonian forest. American Journal of Tropical Medicine and Hygiene, 25(5): 671-674.

MILES, M.A.; DE SOUZA, A.A. \& POVOA, M.M. 1981. Mammal traking and nest location in Brazilian forest with an improved spool-and-line device. Journal of Zoology, 195: 331-347.

MONTEIRO-FILHO, E.L.A. \& DIAS, V.S. 1990. Observações sobre a biologia de Lutreolina crassicaudata (Mammalia: Marsupialia). Revista Brasileira de Biologia, 50(2): 393-399. 
MONTEIRO-FILHO, E.L.A. \& MACHADO, L.O.M. 1996. The utilization of nest-boxes by small mammals. Ciência $e$ Cultura, 48(4): 221-224.

MORAES JÚNIOR, E.A. \& CHIARELLO, A.G. 2005a. A radio tracking study of home range and movements of the marsupial Micoureus demerarae (Thomas) (Mammalia, Didelphidae). Revista Brasileira de Zoologia, 22(1): 85-91.

MORAES JÚNIOR, E.A. \& CHIARELLO, A.G. 2005b. Sleeping sites of the woolly mouse opossum Micoureus demerarae (Thomas) (Didelphimorphia, Didelphidae) in the Atlantic Forest of south-eastern Brazil. Revista Brasileira de Zoologia, 22(4): 839-843.

MOURA, M.C.; CAPARELLI, A.C.; FREITAS, S.R. \& VIEIRA, M.V. 2005. Scale-dependent habitat selection in three didelphid marsupials using the spool-and-line technique in the Atlantic Forest of Brazil. Journal of Tropical Ecology, 21: 337-342.

NITIKMAN, L.Z. \& MARES, M.A. 1987. Ecology of small mammals in a gallery forest of Central Brazil. Annals of Carnegie Museum, 56: 75-95.

OLIVEIRA P.S \& RATTER, J. 2002. Vegetation physiognomies and woody flora of the Cerrado biome. Pp 91-120. In P.S Oliveira \& R.J. Marquis (eds). The Cerrados of Brazil: Ecology and natural history of a neotropical savanna. Columbia University Press. New York. 424p.

PAGLIA, A.P.; DE MARCO Jr, P.; COSTA, F.M.; PEREIRA, R.F. \& LESSA, G. 1995. Heterogeneidade estrutural e diversidade de pequenos mamíferos em um fragmento de mata secundária de Minas Gerais, Brasil. Revista Brasileira de Zoologia, 12(1): 67-79.

PASSAMANI, M. 1995. Vertical stratification of small mammals in Atlantic hill forest. Mammalia, 59: 276-279.

PIANKA, E.R. 1999. Evolutionary ecology. Sixth edition. Addison-Wesley Press, Boston. 512p.

PIRES, A.S.; LIRA, P.K.; FERNANDEZ, F.A.S.; SCHITTINI, G.M. \& OLIVEIRA, L.C. 2002. Frequency of movements of small mammals among Atlantic Coastal Forest fragments in Brazil. Biological Conservation, 108: 229-237.

PIRES, A.S.; FERNANDEZ, F.A.S.; FREITAS, D. \& FELICIANO, B.R. 2005. Influence of edge and fire-induced changes on spatial distribution of small mammals in Brazilian Atlantic Forest fragments. Studies on Neotropical Fauna and Environment, 40: 7-14.

PIRES, A.S. \& FERNANDEZ, F.A.S. 1999. Use of space by the marsupial Micoureus demerarae in small Atlantic Forest in south-eastern Brazil. Journal of Tropical Ecology, 15: 279-290.

PIRES, A.S.; FERNANDEZ, F.A.S. \& FREITAS, D. 1999.
Patterns of space use by Micoureus demerarae (Marsupialia: Didelphidae) in a fragment of Atlantic forest in Brazil. Mastozoologia Neotropical, 6: 39-45.

PREVEDELLO, J.A.; FERREIRA, P.; PAPI, B.S.; LORETTO, D. \& VIEIRA, M.V. 2008. Uso do espaço vertical por pequenos mamíferos no Parque Nacional Serra dos Órgãos, RJ: um estudo de 10 anos utilizando três métodos de amostragem. Revista Espaço \& Geografia, 11: 95-119.

RIZZINI, C.T. 1979. Tratado de fitogeografia do Brasil. Aspectos sociológicos e florísticas. Segundo volume. EDUSP/ HUCITEC. São Paulo. 374p.

SCHOENER, T.W. 1974. Resource partioning in ecological communities. Science, 185 (1): 27-39.

SPENCER, S.R.; CAMERON, G.N. \& SWIHART, R.K. 1990. Operationally defining home range: temporal dependence exhibited by hispid cotton rats. Ecology, 71: 1817-1822.

STREILEIN, K.E. 1982. Ecology of small mammals in the semiarid brazilian caatinga. IV. Habitat selection. Annals of the Carnegie Museum, 51: 331-343.

TUBELIS, D.P. 2000. Aspects on the breeding biology of the gracile mouse opossum Gracilinanus microtarsus in a second growth Forest in southeastern Brazil. Papéis Avulsos de Zoologia, 41(11): 173-185.

UMETSU, F. \& PARDINI, R. 2007. Small mammals in a mosaic of forest remnants and anthropogenic habitats evaluating matrix quality in an Atlantic forest landscape. Landscape Ecology, 22: 517-530.

VALENZUELA, D. \& CEBALLOS, G. 2000. Habitat selection, home range, and activity of the white-nosed coati (Nasua narica) in a Mexican tropical dry forest. Journal of Mammalogy, 81: 810-819.

VIEIRA, E.M. 2006. Padrões de uso vertical do hábitat por marsupiais brasileiros. pp. 217-228 In: N.C. Cáceres \& E.L.A. Monteiro-Filho (eds). Os Marsupiais do Brasil: Biologia, Ecologia e Evolução. Editora da UFMS, Campo Grande. 364p.

VIEIRA, E.M.; IOB, G.; BRIANI, D.C. \& PALMA, A.R.T. 2005. Microhabitat selection and daily movements of two rodents (Necromys lasiurus and Oryzomys scotti) in Brazilian Cerrado, as revealed by a spool-and-line device. Mammalian Biology, 70(6): 359-365.

VIEIRA, E.M. \& MONTEIRO-FILHO, E.L.A. 2003. Vertical stratification of small mammals in the Atlantic rain forest of south-eastern Brazil. Journal of Tropical Ecology, 19: 501-507.

VIEIRA, E.M. \& MARINHO-FILHO, J. 1998. Pre and post-fire habitat utilization by rodents of cerrado from central Brazil. Biotropica, 30(3): 491-496, 
VIEIRA, M.V. 2003. Seasonal niche dynamics in coexisting rodents of the Brazilian Cerrado. Studies on Neotropical Fauna and Environment, 38: 7-15.

VIEIRA, M.V. \& CUNHA, A.A. 2008. Scaling body mass and use of space in three species of marsupials in the Atlantic Forest of Brazil. Austral Ecology, 33: 872-879.

Submetido em 09/07/2008. Aceito em 23/09/2008. 